\title{
Utilização do Pó de Basalto em Substratos para Mudas de Eucalyptus grandis
}

\author{
Tiago Ehlers ${ }^{1}$, Guilherme Oliveira Santos Ferraz de Arruda ${ }^{1}$ \\ ${ }^{1}$ Curso de Engenharia Florestal, Universidade do Oeste de Santa Catarina - UNOESC, Xanxerê/SC, Brasil
}

\section{RESUMO}

O pó de rocha basáltica é um resíduo de mineração com potencial para ser reutilizado como um componente de substratos florestais, por conter elementos minerais importantes, como os macronutrientes. O objetivo deste estudo foi avaliar os efeitos do pó de basalto, adicionado em diferentes dosagens a misturas com vermiculita e composto comercial de turfa, no desenvolvimento de mudas de Eucalyptus grandis W. Hill ex Maiden, aos 180 dias após a semeadura. Analisados os dados morfológicos indicadores de qualidade de mudas florestais - como altura da parte aérea $(h)$, diâmetro do coleto $(d)$ e relação entre altura da parte aérea e diâmetro do coleto $(h / d)$-, concluiu-se que o pó de basalto, em dosagens de 10 a $20 \%$, adicionado a substratos com misturas de vermiculita e composto à base de turfa, é um componente com potencial favorável ao desenvolvimento da altura da parte aérea e do diâmetro do coleto, nas mudas de Eucalyptus grandis. Observou-se também a emergência da parte aérea e a sobrevivência das mudas, cujo percentual, para ambas, foi 100\%.

Palavras-chave: resíduo, rocha basáltica, nutrientes.

\section{Use of Basalt Dust in Substrates for Eucalyptus grandis Seedlings}

\begin{abstract}
Basalt rock dust is a residue of basaltic rock mining with potential to be reused as a component of forestry substrates because of its important mineral elements, such as the macronutrients. This study aimed to evaluate the effects of residual basalt dust, mixed in different strengths with vermiculite and organic compound made from peat, in seedlings of Eucalyptus grandis W. Hill ex Maiden, 180 days after sowing. After analysis of the morphological indicators of quality forest seedlings, such as aerial part height (h), stem diameter (d), and aerial part height/stem diameter ratio $(\mathrm{h} / \mathrm{d})$, it was possible to conclude that basalt dust, in doses of 10 to $20 \%$ added to the substrates with mixtures of vermiculite and peat compost, is a component with favorable potential to the development of aerial part height and stem diameter in Eucalyptus grandis seedlings. It was also possible to observe that both the emergence of the aerial part and the seedling survival reached $100 \%$.
\end{abstract}

Keywords: residue, basaltic rock, nutrients. 


\section{INTRODUÇÃO}

A expansão de florestas plantadas com espécies exóticas comerciais, como as do gênero Eucalyptus, tenta suprir a crescente demanda de matéria-prima para celulose e papel, energia, serraria, laminação, construção civil, óleos essenciais e outros.

Em 2011, o Brasil totalizou 6,5 milhões de hectares de florestas plantadas, dos quais 4,8 milhões de hectares com Eucalyptus spp (ABRAF, 2012). A qualidade das mudas é um fator fundamental para o sucesso dessas florestas, proporcionando rapidez no crescimento e resistência, características decisivas para esse sucesso (Cruz et al., 2004).

Quanto à qualidade, as mudas florestais podem ser avaliadas por meio de parâmetros morfológicos e fisiológicos, sendo os morfológicos os mais utilizados para determinar um padrão, por terem melhor compreensão intuitiva pelos viveiristas (Gomes et al., 2002); dentre tais parâmetros, citam-se a altura da parte aérea, o diâmetro do coleto e a relação entre a altura e o diâmetro. Os atributos morfológicos são determinados de forma visual ou física e, conforme Fonseca (2000), pesquisas têm sido realizadas para mostrar a importância de critérios qualitativos para o melhor desempenho das mudas no campo, após o plantio.

Na produção de mudas com qualidade, o substrato é elemento importante, pois suas características físico-químicas devem favorecer o desenvolvimento da muda. Devido ao reduzido volume do recipiente e à lixiviação de nutrientes nas irrigações de viveiro, a adubação em cobertura tornou-se necessária para as mudas (Maeda et al., 2007; Gonçalves et al., 2000). Tal demanda de nutrientes eleva o custo, mas estimula novas pesquisas com materiais alternativos para adubação (Knapik \& Angelo, 2007) e para substratos. Segundo Stappe \& Balloni (1988), insumos não convencionais tornam-se atraentes pelo baixo custo e por melhorar as propriedades do solo e aumentar a produção vegetal, como é o caso do pó de rochas basálticas. Para Theodoro et al. (2006), este é rico em macronutrientes e, quando disponível na região de uso, sem contaminantes, pode ser incorporado a materiais orgânicos e tornar-se opção de insumo. Rochas basálticas possuem minerais intemperizáveis e ricos em cátions, como feldspatos cálcio-sódicos e piroxênios (Resende et al., 2002), potencializando o uso de seu pó em substratos.

A facilidade de obtê-lo em pedreiras vulcânicas por todo o Brasil o torna mais barato do que outros produtos para adubação (Kiehl, 2002). Para Theodoro (2000), isso possibilita aplicá-lo em solos com custo quase 20 vezes menor do que o de insumos convencionais. Pesquisas sobre o efeito dos pós de rochas na microbiota do solo e de seu acúmulo revelam o potencial do pó de basalto para uso em solos (Suguino et al., 2011) e para compor substratos para mudas, quando contribuirá para reduzir seu volume em extensões de terra (Nolasco et al., 2000). Para Theodoro \& Leonardos (2006), o uso do pó de rocha vulcânica em solo arenoso aumenta o $\mathrm{pH}$ e os teores de $\mathrm{Ca}, \mathrm{Mg}, \mathrm{P}$ e K, podendo estes permanecer maiores até mesmo por cinco anos. Com o pó de basalto aplicado no solo, obtêm-se respostas positivas para a elevação do $\mathrm{pH}$ e dos teores de $\mathrm{Mg}$ e Si do solo, assim como para a nutrição de mudas de Eucalyptus benthamii (Silva et al., 2012). O objetivo deste trabalho foi avaliar os efeitos do pó de basalto, adicionado em diferentes dosagens em misturas com vermiculita e composto comercial de turfa, no desenvolvimento morfológico de mudas de Eucalyptus grandis.

\section{MATERIAL E MÉTODOS}

O experimento foi conduzido de novembro de 2011 a maio de 2012, em casa de vegetação não climatizada da Universidade do Oeste de Santa Catarina, em Xanxerê-SC, a $26^{\circ} 52^{\prime} 37^{\prime \prime}$ de latitude $S, 52^{\circ} 24^{\prime} 15^{\prime \prime}$ de longitude $\mathrm{W}$ e altitude de $800 \mathrm{~m}$. O clima do local é do tipo Cfa (Koeppen), subtropical (mesotérmico úmido com verão quente), com temperatura média anual entre 16 e $17^{\circ} \mathrm{C}$ e precipitação média de 2.100 a $2.300 \mathrm{~mm} /$ ano (Santa Catarina, 2003).

Foram utilizadas sementes peletizadas de Eucalyptus grandis, adquiridas na empresa MP Administradora Florestal Ltda; serviram, como recipientes, tubetes cônicos estriados de polipropileno com capacidade de $100 \mathrm{~cm}^{3}$.

Para formular os tratamentos, utilizaram-se três componentes em misturas: pó de rocha basáltica, vermiculita expandida comercial com granulometria 
fina Terra Mater ${ }^{\circledast}$ e composto comercial turfoso Turfa Fértil ${ }^{\circledast}$, que foram homogeneizados em sacos de ráfia para cada tratamento (Tabela 1 ).

Adotou-se o delineamento inteiramente casualizado, com cinco tratamentos, quatro repetições e 12 tubetes por repetição. A vermiculita e o composto à base de turfa compuseram o substrato juntamente com o pó de basalto, por serem produtos amplamente utilizados na produção comercial de mudas florestais, com características físicas e químicas favoráveis ao seu desenvolvimento em tubetes.

A vermiculita Terra Mater $^{\circledast}$ contém o mineral expandido inerte, não tóxico, poroso, farelado com granulometria fina e capacidade de retenção de água (CRA) mínima de 200\% em massa. O composto Turfa Fértil ${ }^{\circledR}$, além da turfa, contém casca de Pinus compostada, calcário e aditivo fertilizante; possui densidade de $235 \mathrm{~kg} / \mathrm{m}^{3}$ em base seca, $\mathrm{pH}$ 5,5 \pm 0,2, umidade máxima de $55 \%$ e CRA de $57 \%$. O pó de basalto, obtido em pedreira ativa da empresa Planaterra, em Guatambu-SC, teve análise física de rotina realizada em laboratório da empresa e apresentou $91,6 \%$ do material seco com granulometria menor do que $0,07 \mathrm{~mm}$ (passando pela peneira ASTM n. ${ }^{\circ}$ 200), densidade aparente (D.A.) do grão seco de $1,526 \mathrm{~kg} / \mathrm{cm}^{3}$, densidade real (D.R.) do grão seco de $2,923 \mathrm{~kg} / \mathrm{cm}^{3}$ e equivalente de areia (E.A.) de $55,4 \%$. O pH, a umidade e os macronutrientes do pó de basalto foram analisados no Laboratório de Solos da Empresa de Pesquisa Agropecuária e Extensão
Rural de Santa Catarina - EPAGRI, em Chapecó-SC (Tabela 2).

Os tubetes, preenchidos com os tratamentos, foram colocados em mesas metálicas quadriculadas com tela de arame a $0,80 \mathrm{~m}$ de altura do solo, nas posições de cada repetição definidas por sorteio. Umedeceu-se o substrato por aspersão durante dois minutos e realizou-se a semeadura manual, com uma semente peletizada por tubete. O programa de irrigação automática consistiu de cinco aspersões diárias de dois minutos cada, intervaladas a cada três horas, com início às $7 \mathrm{~h}$.

Os parâmetros morfológicos escolhidos para avaliar o desenvolvimento das mudas, em resposta aos tratamentos com pó de basalto nos substratos, foram: altura da parte aérea $(h)$, diâmetro do coleto (d) e a relação altura/diâmetro $(h / d)$, recomendados por Knapik (2005), Carneiro (1995), McTague \& Tinus (1996) e Johnson \& Cline (1991). Decorridos 60 dias da semeadura, iniciou-se a coleta de dados parciais, repetindo-a aos 90, 120 e 150 dias, para fins de acompanhamento. Aos 180 dias, fez-se a última coleta de dados, sendo que somente as médias de $h, d$ e $h / d$ desta coleta foram utilizadas para análise estatística.

Para determinação das alturas da parte aérea das mudas, mediu-se do nível do substrato até a ponta da última folha, utilizando-se régua graduada em centímetros; para os diâmetros do coleto, mediu-se no nível do substrato, utilizando-se paquímetro digital

Tabela 1. Composição dos tratamentos utilizados neste estudo.

Table 1. Treatments composition used in this study.

\begin{tabular}{ccc} 
Tratamento & \multicolumn{1}{c}{ Composição } & Representação \\
T1 & 0\% pó de basalto; $10 \%$ vermiculita e $90 \%$ composto turfoso & $(0: 10: 90)$ \\
T2 & 10\% pó de basalto; $10 \%$ vermiculita e $80 \%$ composto turfoso & $(10: 10: 80)$ \\
T3 & 20\% pó de basalto; $10 \%$ vermiculita e $70 \%$ composto turfoso & $(20: 10: 70)$ \\
T4 & 30\% pó de basalto; $10 \%$ vermiculita e $60 \%$ composto turfoso & $(30: 10: 60)$ \\
T5 & 40\% pó de basalto; $10 \%$ vermiculita e 50\% composto turfoso & $(40: 50)$ \\
\hline
\end{tabular}

Tabela 2. Relatório da análise do pó de basalto para $\mathrm{pH}$, umidade e macronutrientes.

Table 2. Analysis report of basalt powder used for $\mathrm{pH}$, moisture and macronutrients.

\begin{tabular}{|c|c|c|c|c|c|c|c|c|}
\hline \multirow{2}{*}{ Material } & \multicolumn{8}{|c|}{$(\%)$} \\
\hline & $\mathbf{p H}^{* *}$ & Umidade $\left(\right.$ a $\left.65^{\circ} \mathrm{C}\right)$ & $\mathbf{N}^{*}$ & $\mathbf{P}_{2} \mathbf{O}_{5}^{\star a}$ & $\mathbf{P}_{2} \mathbf{O}_{5}^{* * * *}$ & $\mathbf{K}_{2} \mathbf{O}^{* * *}$ & $\mathrm{Ca}^{\star}$ & $\mathbf{M g}^{\star}$ \\
\hline Pó de basalto & 8,0 & 2,30 & 0,77 & 0,16 & 0,00 & 0,18 & 0,60 & 0,08 \\
\hline
\end{tabular}


Digimess $^{\circledast}$ com leitura em milímetros. Com a simples divisão entre estes valores de altura e diâmetro, obteve-se o valor do parâmetro $h / d$, representado sem unidade. Os dados médios obtidos aos 180 dias foram submetidos à análise estatística pelo teste de Bartlett e, após comprovada a homogeneidade das variâncias, fez-se a análise de variância (ANOVA), além da comparação de médias (Teste de Tukey) a 5\% de probabilidade, com auxílio do software Assistat versão 7.5. Acompanhou-se também o processo germinativo diariamente, registrando-se a emissão da parte aérea e o desenvolvimento das plântulas em cada tubete, para, ao final do experimento, obter-se o percentual de sobrevivência nos tratamentos.
Para auxiliar na avaliação do desempenho dos três parâmetros por tratamento, elaborou-se uma pontuação para as médias de $h, d$ e $h / d$ obtidas aos 180 dias. Atribuiu-se 5, 4, 3, 2 e 1 ponto, do maior ao menor valor de cada parâmetro, e a soma de pontos dos tratamentos serviu como indicativo do grau de contribuição destes para cada parâmetro.

\section{RESULTADOS E DISCUSSÃO}

As médias gerais dos três parâmetros, calculadas após cada medição, foram representadas com o intuito de visualizar graficamente suas evoluções (Figura 1).
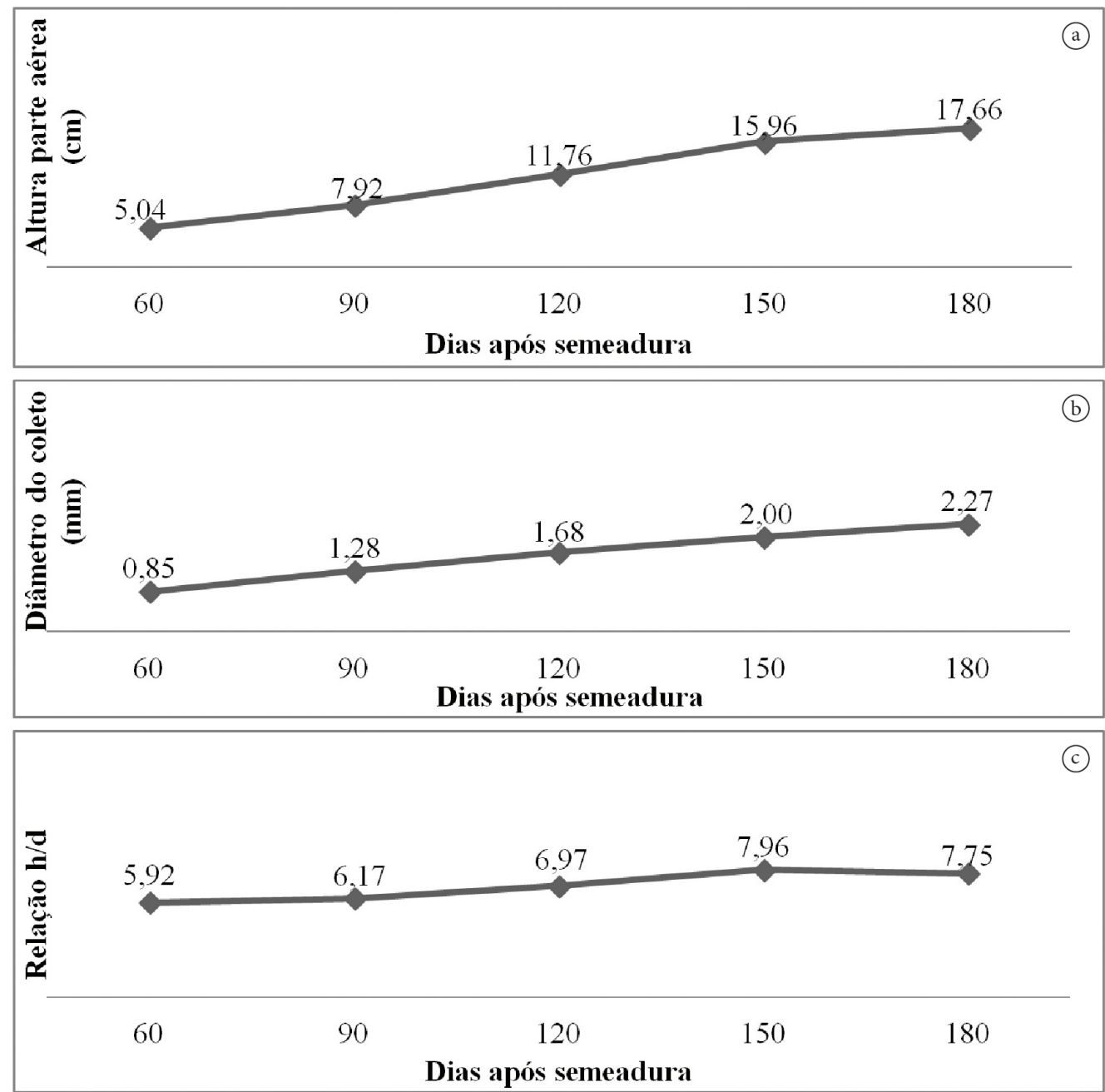

Figura 1. Médias gerais ao longo do estudo: a- altura da parte aérea; b- diâmetro do coleto; c- relação $h / d$ das mudas aos 60, 90, 120, 150 e 180 dias após a semeadura.

Figure 1. General average during the study: a- aerial part height; b- stem diameter; c- ratio $h / d$ of seedlings at 60 , $90,120,150$ and 180 days after sowing. 
Verificou-se que as médias gerais de altura da parte aérea e de diâmetro do coleto foram crescentes até os 180 dias, e que os maiores incrementos percentuais ocorreram dos 60 dias para os 90 dias, para ambos os parâmetros. Analisando-se os incrementos das médias gerais, em percentagem, ao longo do experimento, verificou-se que, para as alturas, houve incremento de $57,27 \%$ dos 60 dias para 90 dias; de 48,40\% dos 90 dias para 120 dias; de $35,71 \%$ dos 120 dias para 150 dias, e de 10,63\% dos 150 dias para 180 dias.

Para os diâmetros dos coletos, os incrementos foram de 50,75\% dos 60 para 90 dias; de 31,13\% dos 90 para 120 dias; de $18,94 \%$ dos 120 para 150 dias, e de $13,60 \%$ dos 150 para 180 dias.

Para a relação $h / d$, houve incremento nos valores de $4,32 \%$ dos 60 para 90 dias; de $12,95 \%$ dos 90 para 120 dias, e de $14,10 \%$ dos 120 para 150 dias. Dos 150 para os 180 dias, houve diminuição deste quociente em 2,61\%, indicando maior desenvolvimento da muda em diâmetro do que em altura neste período.

Reis (2006), em pesquisa sobre a variação espacial e temporal de parâmetros morfológicos de mudas de Eucalyptus grandis para expedição do viveiro, obteve, aos 115 dias, com substrato comercial Plantmax (turfa, vermiculita, casca de Pinus e carvão moído), média de altura da parte aérea entre 14,57 e 20,81 $\mathrm{cm}$, de diâmetro do coleto entre 1,81 e 2,08 mm e, de relação $h / d$, médias entre 7,81 e 10,32. Observandose os valores das médias gerais deste estudo aos 120 dias (Figura 1), verifica-se que Reis (2006) obteve, com cinco dias a menos, valores maiores em $23,89 \%$, $12,42 \%$ e $12,05 \%$, respectivamente, para $h, d$ e $h / d$, influenciados pelas características nutricionais e físicas do substrato.

Os resultados estatísticos de comparação das médias obtidas aos 180 dias, para $h, d$ e $h / d$ em cada tratamento (Tukey a $5 \%$ de probabilidade), foram sumarizados na Tabela 3.

Escolheu-se a altura da parte aérea da muda para ser analisada devido à sua fácil medição, por não danificar a muda e por ser considerada um excelente parâmetro qualitativo, conforme Knapik (2005). O tratamento 3 (20\% PB, 10\% VE; $70 \% \mathrm{TF)}$ obteve o maior valor médio para altura das mudas $(19,60 \mathrm{~cm})$, seguido pelos tratamentos $2(19,17 \mathrm{~cm}) \mathrm{e}$ $4(18,34 \mathrm{~cm})$ (Tabela 3$)$.

No entanto, não houve diferença significativa entre as alturas dos tratamentos 3, 2 e 4 , sendo $20 \%$ $\mathrm{PB}, 10 \% \mathrm{~PB}$ e $30 \% \mathrm{~PB}$, respectivamente, e entre as médias dos tratamentos 5 e 4 , com respectivos $40 \%$ e $30 \%$ PB (Tabela 3). Os tratamentos 3, 2 e 4, com as melhores alturas, apresentaram diferença significativa em relação à média do tratamento 1 (testemunha), indicando terem contribuído para o incremento da altura nas mudas. Observou-se que o tratamento $5(40 \% \mathrm{~PB})$ e o tratamento 1 , com a menor média deste estudo, não diferiram significativamente entre si na altura da parte aérea (Tabela 3). Este resultado é indicativo de que o tratamento 5 não contribuiu para o incremento da altura, possivelmente devido a menor porosidade e menor aeração no substrato, geradas pela maior quantidade do pó de basalto neste tratamento. Os $40 \%$ de pó de basalto do tratamento 5 podem ter causado efeito cimentante devido à granulometria pequena de suas partículas

Tabela 3. Resultados do teste estatístico comparativo de médias (Tukey a 5\% de probabilidade) para altura da parte aérea $(h)$, diâmetro do coleto $(d)$ e relação $h / d$ das mudas nos tratamentos, aos 180 dias.

Table 3. Results of statistical comparative test of averages (Tukey test at $5 \%$ probability) for aerial part height $(h)$, stem diameter $(d)$ and ratio $h / d$ of seedlings in the treatments, at 180 days.

\begin{tabular}{ccccc}
\multirow{2}{*}{ Tratamento } & $\begin{array}{c}\text { Composição } \\
(\text { PB: VE: TF) }\end{array}$ & $\begin{array}{c}\text { Altura da parte aérea } \\
(h)\end{array}$ & $\begin{array}{c}\text { Diâmetro do coleto } \\
(d)\end{array}$ & \multirow{2}{*}{ Relação $h / d$} \\
\cline { 2 - 4 } & $(\%)$ & $(\mathbf{c m})$ & $(\mathbf{m m})$ & $6,80 \mathrm{~b}$ \\
\hline 1 & $(00: 10: 90)$ & $15,32 \mathrm{c}$ & $2,25 \mathrm{~b}$ & $7,98 \mathrm{ab}$ \\
\hline 3 & $(10: 10: 80)$ & $19,17 \mathrm{a}$ & $2,40 \mathrm{ab}$ & $7,96 \mathrm{ab}$ \\
\hline 5 & $(20: 10: 70)$ & $19,60 \mathrm{a}$ & $2,46 \mathrm{a}$ & $8,05 \mathrm{a}$ \\
\hline
\end{tabular}

Médias seguidas de pelo menos uma letra igual dentro da coluna não diferem entre si pelo Teste de Tukey a 5\% de probabilidade. PB: Pó de basalto; VE: Vermiculita expandida; TF: composto comercial Turfa Fértil ${ }^{\oplus}$. 
(< 0,4 mm), ratificando informações de Knapik \& Angelo (2007). Para Schmitz et al. (2002), este efeito pode ser resultado da retenção de água pelas partículas e da redução do espaço de aeração ou, de acordo com Kämpf (2000), do fechamento dos poros e da compactação do material.

Ao pesquisarem combinações de turfa e terra de subsolo com bagaço de cana, casca de arroz carbonizada, folhas e galhos de eucalipto e vermiculita, para substrato de mudas de Eucalyptus grandis para tubetes, Aguiar et al. (1989) obtiveram, aos 90 dias após a semeadura, média de 20,2 cm para altura da parte aérea, valor expressivamente maior do que a média geral de 7,92 cm deste estudo, na mesma idade (Figura 1a), resultante provável das melhores condições físicas e minerais destas combinações, em termos de retenção de água, consistência de substrato, fixação de nutrientes e teor de matéria orgânica. Gomes et al. (2003), ao analisarem altura de mudas de Eucalyptus grandis, em diferentes dimensões de tubetes e com fertilização de NPK, verificaram que, aos 120 dias, as médias de altura da parte aérea variaram de 21,62 a $55,01 \mathrm{~cm}$ em tubetes $10 \%$ maiores em volume que os deste estudo, configurando resultados numéricos também superiores à média geral de $11,76 \mathrm{~cm}$, na mesma idade (Figura 1a).

O diâmetro do coleto foi escolhido para análise baseado em informações de Carneiro (1995), para o qual este parâmetro pode indicar se há equilíbrio no crescimento, e de McTague \& Tinus (1996), que o consideram mais ajustado para predizer a sobrevivência das mudas no campo. Embora o tratamento $3(20 \% \mathrm{~PB})$ tenha apresentado valor médio maior para diâmetro do coleto $(2,46 \mathrm{~mm})$, não existiu diferença significativa entre os diâmetros médios das mudas obtidas com os tratamentos 3 e 2 , com $20 \%$ PB e $10 \%$ PB, respectivamente; e, da mesma forma, entre os diâmetros médios dos tratamentos 2, 4 e 1, com respectivos $10 \% \mathrm{~PB}, 30 \% \mathrm{~PB}$ e $0 \% \mathrm{~PB}$. (Tabela 3).

Observa-se, pela Tabela 3, que os tratamentos 3 e 2 , com respectivos $20 \% \mathrm{~PB}$ e $10 \% \mathrm{~PB}$, apresentaram diferença significativa em relação ao tratamento 1 (testemunha), indicando que contribuíram para o aumento do diâmetro do coleto das mudas neste estudo. Os tratamentos 2 e 4, com 10\% PB e 30\% PB, respectivamente, por não diferirem estatisticamente do tratamento 1 , não contribuíram no incremento do diâmetro. O tratamento $5(40 \% \mathrm{~PB})$, com a menor média para diâmetro do coleto, inferior inclusive à do tratamento 1 (testemunha), mostra sua ineficiência em relação a esse parâmetro. Lopes (2005), ao pesquisar a qualidade de mudas florestais com idade de 90 dias, obteve diâmetros de coleto de 2,07 a 4,41 $\mathrm{mm}$ em Eucalyptus urophylla e de 2,07 a 3,69 mm em Eucalyptus camaldulensis, utilizando substrato comercial da Eucatex Agro $^{\circledR}$ (turfa, vermiculita expandida e casca de Pinus). Aguiar et al. (1989), em estudo sobre composição de substratos para mudas de Eucalyptus spp em tubetes, obtiveram, aos 90 dias, diâmetros de coleto entre 2,1 e 2,4 mm. Estes valores de coleto são maiores do que a média geral deste estudo com Eucalyptus grandis, na mesma idade $(1,28 \mathrm{~mm})$ (Figura 1b), e reforçam que, além do fator espécie, as características físicas e nutricionais do substrato também influenciam neste parâmetro.

O parâmetro $h / d$, ou quociente de robustez, foi escolhido com base em estudos de Johnson \& Cline (1991), Knapik (2005) e Carneiro (1995), para os quais esse parâmetro indica o quanto delgada está a muda, a capacidade de ela estabelecer-se após o plantio e avalia que valores, entre 5,4 a 8,1 , são bons e apropriados para as mudas de eucalipto.

Os resultados da Tabela 3 demonstraram que o tratamento 4 (30\% PB) obteve o maior valor para a relação $h / d$ das mudas $(8,05)$. No entanto, indicou-se que não houve diferença significativa entre as médias deste parâmetro para os tratamentos $4,2,3$ e 5, com $30 \% \mathrm{~PB}, 10 \% \mathrm{~PB}, 20 \% \mathrm{~PB}$ e $40 \% \mathrm{~PB}$, respectivamente. Também não existiu diferença significativa entre os tratamentos 2, 3 e 5, com $10 \%$ PB, 20\% PB e $40 \%$ PB, respectivamente, em relação à média do tratamento 1 (0\% PB ou testemunha). Somente a média do tratamento $4(30 \% \mathrm{~PB})$ diferiu significativamente da média do tratamento 1 , indicando contribuição no incremento de $h / d$ das mudas.

Ao contrário dos parâmetros 'altura da parte aérea' e 'diâmetro do coleto', quanto menor for o valor da relação $h / d$, melhor será para a muda, demonstrando maior equilíbrio entre ambos os parâmetros e tornando maior a robustez da muda. Lopes (2005) obteve valores para $h / d$ entre 7,76 e 9,62 para Eucalyptus urophylla, e entre 8,98 e 9,76 
Tabela 4. Resultados da pontuação para os tratamentos, com base no desempenho dos parâmetros $h, d$ e $h / d$ aos 180 dias após a semeadura.

Table 4. Score results for the treatments, based on performance of $h, d$ and $\mathrm{h} / \mathrm{d}$ parameters at180 days after sowing.

\begin{tabular}{ccccc} 
Tratamento (\%PB: \%VE: \% TF) & \multicolumn{3}{c|}{ Pontuação } \\
\cline { 2 - 5 } & h & d & h/d & Total \\
\hline T1 (00:10:90) & 1 & 2 & 1 & 4 \\
T2 (10:10:80) & 4 & 4 & 4 & 12 \\
T3 (20:10:70) & 5 & 5 & 3 & 11 \\
T4 (30:10:60) & 3 & 3 & 5 & 5 \\
\hline T5 (40:10:50) & 2 & 1 & 2 & \\
\hline
\end{tabular}

PB: Pó de basalto; VE: Vermiculita expandida; TF: composto comercial Turfa Fértil ${ }^{\circ} ; h$ : altura da parte aérea da muda; $d$ : diâmetro do coleto da muda; $h / d$ : relação entre ambos os parâmetros.

para Eucalyptus camaldulensis aos 90 dias após a semeadura, que são numericamente maiores do que o valor 6,17 obtido para o parâmetro neste estudo, na mesma idade (Figura 1c).

O critério de pontos para auxiliar na avaliação do desempenho dos parâmetros nos tratamentos demonstrou que o tratamento 3 (20\% PB; $10 \% \mathrm{VE}$; $70 \% \mathrm{TF}$ ) obteve a maior pontuação total (Tabela 4), destacando-se pela maior contribuição no desempenho da altura da parte aérea e no diâmetro do coleto, seguido pelo tratamento 2 (10\% PB; $10 \%$ $\mathrm{VE}$; $80 \% \mathrm{TF}$ ). O tratamento 1 ( $0 \% \mathrm{~PB} ; 10 \% \mathrm{VE}$; 90\% TF) e o tratamento 5 ( $40 \%$ PB; $10 \%$ VE; $50 \%$ $\mathrm{TF}$ ) obtiveram as menores pontuações totais, sendo um indicativo de pouca influência no desempenho da altura e do diâmetro do coleto; demonstra-se, dessa forma, ser importante e necessário adequar as quantidades do pó de basalto em substratos para mudas de Eucalyptus grandis.

\section{CONCLUSÕES}

O pó de basalto, em dosagens de 10 a $20 \%$, adicionado em substratos com misturas de vermiculita e composto à base de turfa, é um componente com potencial favorável para uso na produção de mudas de Eucalyptus grandis.

\section{STATUS DA SUBMISSÃO}

Recebido: 24/02/2013

Aceito: 06/01/2014

Publicado: 31/02/2014

\section{AUTOR(ES) PARA CORRESPONDÊNCIA}

\section{Guilherme Oliveira Santos Ferraz de Arruda}

Curso de Engenharia Florestal, Universidade do

Oeste de Santa Catarina - UNOESC

CEP 89820-000, Xanxerê, SC, Brasil

e-mail: guilherme.arruda@unoesc.edu.br

\section{REFERÊNCIAS}

Aguiar IB, Valeri SV, Banzatto DA, Corradini L, Alvarenga SF. Seleção de componentes de substrato para produção de mudas de eucalipto em tubetes. Revista IPEF 1989; (41-42): 36-43.

Associação Brasileira de Produtores de Florestas Plantadas - ABRAF. Anuário Estatístico da ABRAF 2012 - Ano Base 2011. [cited 2013 jan. 11]. Available from: http://www.abraflor.org.br/estatisticas/ABRAF12/ ABRAF12-BR.pdf.

Carneiro JGA. Produção e controle de qualidade de mudas florestais. Curitiba: FUPEF; 1995. 451 p.

Cruz CAF, Paiva HN, Gomes KCO, Guerrero CRA. Efeito de diferentes níveis de saturação por bases no desenvolvimento e qualidade de mudas de Ipê Roxo (Tabebuia impetiginosa (Mart.) Standley). Scientia Forestalis, 2004; (66): 100-107.

Fonseca EP. Padrão de qualidade de mudas de Trema micrantha (L.) Blume, Cedrela fissilis Vell. e Aspidosperma polyneuron Müll. Arg. produzidas sob diferentes períodos de sombreamento [tese]. Jaboticabal: Faculdade de Ciências Agrárias e Veterinárias, Universidade Estadual Paulista; 2000.

Gomes JM, Couto L, Leite HG, Xavier A, Garcia SLR. Parâmetros morfológicos na avaliação da qualidade de mudas de Eucalyptus grandis. Revista Árvore 2002; 26(6): 655-664. http://dx.doi.org/10.1590/S010067622002000600002 
Gomes JM, Couto L, Leite HG, Xavier A, Garcia SLR. Crescimento de mudas de Eucalyptus grandis em diferentes tamanhos de tubetes e fertilização N-P-K. Revista Árvore 2003; 27(2): 113-127. http://dx.doi. org/10.1590/S0100-67622003000200001

Gonçalves JLM, Santarelli EG, Moraes SP No, Manara MP. Produção de mudas de espécies nativas: substrato, nutrição, sombreamento e fertilização. In: Gonçalves JLM, Benedetti V, editores. Nutrição e fertilização florestal. Piracicaba: IPEF; 2000.

Johnson JD, Cline ML. Seedling quality of southern pines. In: Duryea ML, Dougherty PM, editors. Forest regeneration manual. Dordrecht: Kluwer Academic Press; 1991. http://dx.doi.org/10.1007/978-94-0113800-0_8

Kämpf AN. Produção comercial de plantas ornamentais. Guaíba: Agropecuária; 2000.

Kiehl EJ. Manual de Compostagem: maturação e qualidade do composto. 3. ed. Piracicaba: Kiehl EJ; 2002.

Knapik JG. Utilização do pó de basalto como alternativa à adubação convencional na produção de mudas de Mimosa scabrella Benth e Prunus sellowii Koehne [dissertação]. Curitiba: Universidade Federal do Paraná; 2005.

Knapik JG, Angelo AC. Pó de basalto e esterco equino na produção de mudas de Prunus sellowii Koehne (Rosaceae). Floresta 2007; 37(3): 427-436.

Lopes ED. Qualidade de mudas de Eucalyptus urophylla, E. camaldulensis e E. citriodora produzidas em blocos prensados e em dois modelos de tubetes e seu desempenho no campo [dissertação]. Vitória da Conquista: Universidade Estadual do Sudoeste da Bahia; 2005.

Maeda S, Dedecek RA, Agostini RB, Andrade GC, Silva HD. Pesquisa Florestal Brasileira 2007; (54): 97-104.

McTague JP, Tinus R. The effects of seedlings quality and forest site weather on field survival of ponderosa pine. Tree Planters' Notes 1996; 47(1): 16-23.

Nolasco AM, Guerrini IA, Benedetti V. Uso de resíduos urbanos e industriais como fontes de nutrientes $\mathrm{e}$ condicionadores do solo em plantios florestais. In: Gonçalves JLM, Benedetti V, editores. Nutrição e fertilização florestal. Piracicaba: Ipef; 2000.

Reis ER. Variação espacial e temporal dos parâmetros morfológicos em mudas de Pinus e Eucalipto [dissertação]. Santa Maria: Universidade Federal de Santa Maria; 2006.

Resende M, Curi N, Resende SB, Corrêa GF. Pedologia: base para distinção de ambientes. 4. ed. Viçosa: Neput; 2002. 338 p.

Santa Catarina. Secretaria de Estado de Planejamento, Orçamento e Gestão. Xanxerê: Caracterização regional; 2003. [cited 2012 dez. 10]. Available from: http://cepa. epagri.sc.gov.br/Publicacoes/diagnostico/XANXERE. pdf.

Schmitz JA, Souza PVD, Kämpf AN. Propriedades químicas e físicas de substratos de origem mineral e orgânica para o cultivo de mudas em recipientes. Ciência Rural 2002; 32(6): 937-944. http://dx.doi.org/10.1590/ S0103-84782002000600005

Silva A, Almeida JA, Schmitt C, Coelho CMM. Avaliação dos efeitos da aplicação de basalto moído na fertilidade do solo e nutrição de Eucalyptus benthamii. Floresta 2012; 42(1): 69-76.

Stappe JL, Balloni EA. O uso de resíduos da indústria de celulose como insumos na produção florestal. Revista IPEF 1988; (40): 33-37.

Suguino E, Jacomini AE, Lazarini AP, Martins NA, Faria AM, Perdoná MJ. Utilização do pó de basalto na agricultura. Pesquisa \& Tecnologia 2011; 8(2): 1-5.

Theodoro SH, Leonardos $\mathrm{OH}$. The use of rocks to improve family agriculture in Brazil. Anais da Academia Brasileira de Ciências 2006; 78: 721-730. http://dx.doi. org/10.1590/S0001-37652006000400008

Theodoro SH, Leonardos O, Rocha EL, Rego KG. Experiências de uso de rochas silicáticas como fonte de nutrientes. Espaço \& Geografia 2006; 9(2): 263-292.

Theodoro SH. Fertilização da terra pela terra: uma alternativa para a sustentabilidade do pequeno produtor rural [tese]. Brasília: Universidade de Brasília; 2000. 